\title{
Exact decomposition of a Gaussian-averaged nonlinear function
}

\author{
P. Kolodner, H. S. Kwok, Jerry G. Black, and Eli Yablonovitch \\ Division of Applied Sciences, Harvard University, Cambridge, Massachusetts 02138
}

Received September 25, 1978

\begin{abstract}
We derive an exact formula for the decomposition of Gaussian spatially averaged experimental data. The true nonlinear functional dependence of a physical parameter on the local light intensity can be obtained.
\end{abstract}

In nonlinear optics we are frequently measuring the functional dependence of a physical parameter on light intensity. This immediately presents a problem, since by their nature real optical beams have a varying transverse intensity profile. Any nonlinear measurement will inherently be different at the center of the beam than it is at the edges. A spatially integrated whole-beam measurement must be decomposed to yield the true functional form of the intensity dependence.

One approach is to create a uniform rectangular transverse intensity profile by passing a Gaussian beam through a narrow spatial aperture. This never works well, since the center of a Gaussian is not perfectly uniform and the sharp edges of the aperture produce near-field diffraction rings. Another approach is to assume some nonlinear functional form and to fit it to the spatially integrated experimental data. This reverse procedure does not necessarily result in a unique answer.

In this Letter we present an exact decomposition equation for Gaussian beams that retrieves the true intensity dependence of a measured parameter. Let the Gaussian beam have the transverse intensity profile

$$
I(r)=I_{0} \exp \left(-\frac{2 r^{2}}{\omega_{0}^{2}}\right)
$$

where $I_{0}$ is the central intensity and $\omega_{0}$ is the Gaussian-beam radius. Consider a physical parameter $f(I)$, such as fluorescence, energy deposition, or molecular dissociation, whose nonlinear dependence on intensity we seek to determine. The measurement inherently averages over the full spatial profile of the beam, including the intense region in the center and the fringes where the intensity drops to zero. The measured function is

$$
f_{G}\left(I_{0}\right)=\frac{2}{\pi \omega_{0}^{2}} \int_{0}^{\infty} f[I(r)] 2 \pi r \mathrm{~d} r,
$$

where the area has been normalized to $\pi \omega_{0}^{2} / 2$, the effective area of a Gaussian beam, and $r$ is the transverse radial position. Noticing that $\mathrm{d} I / I=-\mathrm{d}\left(2 r^{2} / \omega_{0}{ }^{2}\right)$, a change of variables may be made in Eq. (1):

$$
f_{G}\left(I_{0}\right)=\int_{0}^{I_{0}} f(I) \frac{\mathrm{d} I}{I}
$$

Differentiating both sides with respect to $I_{0}$ yields

$$
\frac{\mathrm{d} f_{G}\left(I_{0}\right)}{\mathrm{d} I_{0}}=\frac{f\left(I_{0}\right)}{I_{0}}
$$

Changing dummy variables and rearranging terms gives

$$
f(I)=I \frac{\mathrm{d} f_{G}(I)}{\mathrm{d} I},
$$

which may also be written

$$
f(I)=f_{G}(I) \frac{\mathrm{d} \ln f_{G}(I)}{\mathrm{d} \ln I} .
$$

This means that we can obtain the true decomposed function by multiplying the measured function by its local logarithmic derivative. Only experimentally measured parameters appear on the right-hand side of Eqs. (3) and (4).

Basic formulas (3) and (4) should be used with care. The type of decomposition we are discussing here applies only to additive quantities, such as energy and dissociation yield, which can be integrated as in Eq. (1). On the other hand, there are some nonadditive physical parameters to which the decomposition equations, Eqs. (3) and (4), do not apply. For example, the transmission percentage $T(I)$ through a nonlinear absorber is not an additive property. But we can still decompose it because the transmitted power $I T(I)$ is additive and can be substituted in Eqs. (3) and (4). The decomposition formula becomes

$$
T(I)=T_{G}(I)\left[1+\frac{\mathrm{d} \ln T_{G}(I)}{\mathrm{d} \ln I}\right],
$$

where $T_{G}(I)$ is the transmission percentage of the entire Gaussian beam as a whole. Similarly, while a quantity such as the nonlinear absorption coefficient $\alpha(I)$ is not additive, the transmitted power $I \exp [-\alpha(I) l]$ is. A decomposition formula similar to Eq. (5) can be derived for $\alpha(I)$.

The mathematical formulas derived in this Letter were essential for a series of experiments ${ }^{1}$ on infrared multiphoton dissociation that we are conducting in our laboratory. The relevant independent variable is fluence rather than intensity, but the principles are the same. Formulas (3) and (4) are invaluable for measurements of the nonlinear energy deposition and nonlinear dissociation yield. Of necessity, the inte- 
grated effect of the whole laser beam is experimentally observed. Since the transverse intensity distribution is Gaussian, formulas (3) and (4) convert the measured quantities into the exact nonlinear functional dependence on the local intensity.
* Present address: Lawrence Berkeley Laboratory, University of California, Berkeley, California 94720.

Reference

1. J. G. Black, P. Kolodner, M. J. Shultz, E. Yablonovitch, and N. Bloemgergen, Phys. Rev. A, to be published. 